\title{
A POTENTIAL SUPER-VENUS IN THE KEPLER-69 SYSTEM
}

\author{
Stephen R. KAne ${ }^{1}$, Thomas Barclay ${ }^{2}$, And Dawn M. Gelino ${ }^{1}$ \\ ${ }^{1}$ NASA Exoplanet Science Institute, Caltech, MS 100-22, 770 South Wilson Avenue, Pasadena, CA 91125, USA; skane@ipac.caltech.edu \\ 2 NASA Ames Research Center, M/S 244-30, Moffett Field, CA 94035, USA \\ Received 2013 April 29; accepted 2013 May 8; published 2013 May 30
}

\begin{abstract}
Transiting planets have greatly expanded and diversified the exoplanet field. These planets provide greater access to characterization of exoplanet atmospheres and structure. The Kepler mission has been particularly successful in expanding the exoplanet inventory, even to planets smaller than the Earth. The orbital period sensitivity of the Kepler data is now extending into the habitable zones of their host stars, and several planets larger than the Earth have been found to lie therein. Here we examine one such proposed planet, Kepler-69c. We provide new orbital parameters for this planet and an in-depth analysis of the habitable zone. We find that, even under optimistic conditions, this $1.7 R_{\oplus}$ planet is unlikely to be within the habitable zone of Kepler-69. Furthermore, the planet receives an incident flux of 1.91 times the solar constant, which is similar to that received by Venus. We thus suggest that this planet is likely a super-Venus rather than a super-Earth in terms of atmospheric properties and habitability, and we propose follow-up observations to disentangle the ambiguity.
\end{abstract}

Key words: astrobiology - planetary systems - stars: individual (Kepler-69)

\section{INTRODUCTION}

The discovery of exoplanets has spawned a rapidly growing field of astronomy, both in number and diversity. A major contributor to this growth is the success of the transit technique in detecting exoplanets. This has not only produced a high yield of detections, but has enabled many follow-up and characterization studies of these planets due to the additional information the technique provides, in particular the radius of the planet. The Kepler mission has greatly increased the number of confirmed transiting exoplanets, with several thousand candidate planets awaiting confirmation (Batalha et al. 2013; Borucki et al. 2011a, $2011 b)$. The primary mission goal of Kepler is to detect Earthsize planets within the habitable zone (HZ) of their parent stars (Kopparapu et al. 2013), and thus determine their frequency, which is often denoted by $\eta_{\oplus}$. The frequency of terrestrial planets has already been estimated by several authors (Howard et al. 2012; Petigura et al. 2013) and these occurrence rates further applied to the HZ of FGK stars (Traub 2012) and M stars (Dressing \& Charbonneau 2013; Kopparapu 2013). Additional data will likely need to be acquired before $\eta_{\oplus}$ is a robustly determined quantity. Even so, Kepler data has resulted in several promising HZ planets with radii slightly larger than the Earth (Borucki et al. 2012, 2013).

Along the pathway to determining $\eta_{\oplus}$, Kepler will first provide insights into the frequency of Mercury and Venus analogs, both in terms of planet size and incident flux, which we may refer to as $\eta_{\text {Mercury }}$ and $\eta_{\text {Venus }}$, respectively. The fact that Kepler photometry is of sufficient precision to detect Mercurysize planets was recently demonstrated by Barclay et al. (2013b) who discovered a sub-Mercury size planet in the Kepler-37 system. Since Venus and Earth are approximately the same size, the Venus analogs will emerge earlier than the Earth analogs due to the increased geometric transit probability (Kane \& von Braun 2008). Although it is not possible to directly distinguish between the Venus and Earth analogs in terms of their surface conditions, we can determine whether the planets lie within the $\mathrm{HZ}$ of their stars as well as estimate equilibrium temperatures.

The recent discovery of two planets in the Kepler-69 system by Barclay et al. (2013a) presents another possible case for an
HZ planet, that of Kepler-69c. The estimated radius of the planet is $\sim 1.7 R_{\oplus}$, which places it in the category of a "super-Earth" type of object. The determination of the HZ was conducted based on assumptions regarding planetary albedos and equilibrium temperatures. However, the planet resides on the hot edge of what one would consider a habitable region.

Here we perform a re-analysis of this system to determine the $\mathrm{HZ}$ classification of the planets. In Section 2, we provide a self-consistent maximum likelihood estimate (MLE) Keplerian solution for both planets and discuss their values in relation to the Markov Chain Monte Carlo (MCMC) estimations provided by Barclay et al. (2013a). In Section 3 we calculate HZ regions for the star under various assumptions and show that Kepler69c is unlikely to be an HZ planet. In Section 4 we describe the implications of this result for the planetary evolution and atmosphere and show that this planet is a strong candidate to be considered a super-Venus. We also discuss potential signatures of such a planet which may be used to test the hypothesis.

\section{THE KEPLER-69 ORBITAL SOLUTION}

In order to provide an accurate $\mathrm{HZ}$ analysis, we first require a complete and self-consistent model of the star and the planetary orbits. For the star, we adopt the stellar properties derived by Barclay et al. (2013a). These were extracted from Keck I High Resolution Echelle Spectrometer spectra through both a Spectroscopy Made Easy analysis (Valenti \& Fischer 2005) and a comparison with a library of stellar spectra. The relevant stellar parameters are shown in the left side of Table 1.

The orbital parameters for the planets provided by Barclay et al. (2013a) were estimated using an MCMC algorithm (Foreman-Mackey et al. 2013) with an implicit prior on eccentricity $e$ imposed by the fit parameters of $e \cos \omega$ and $e \sin \omega$, where $\omega$ is the argument of periastron (Eastman et al. 2013). This is corrected for using a $1 / e$ prior on $e$ and the final parameters reported are the median values of the posterior distributions. A prior on the mean stellar density is determined from stellar evolution models using the spectroscopic parameters. The parameters resulting from the MCMC analysis are shown in Table 1. A problem with this approach of reporting 

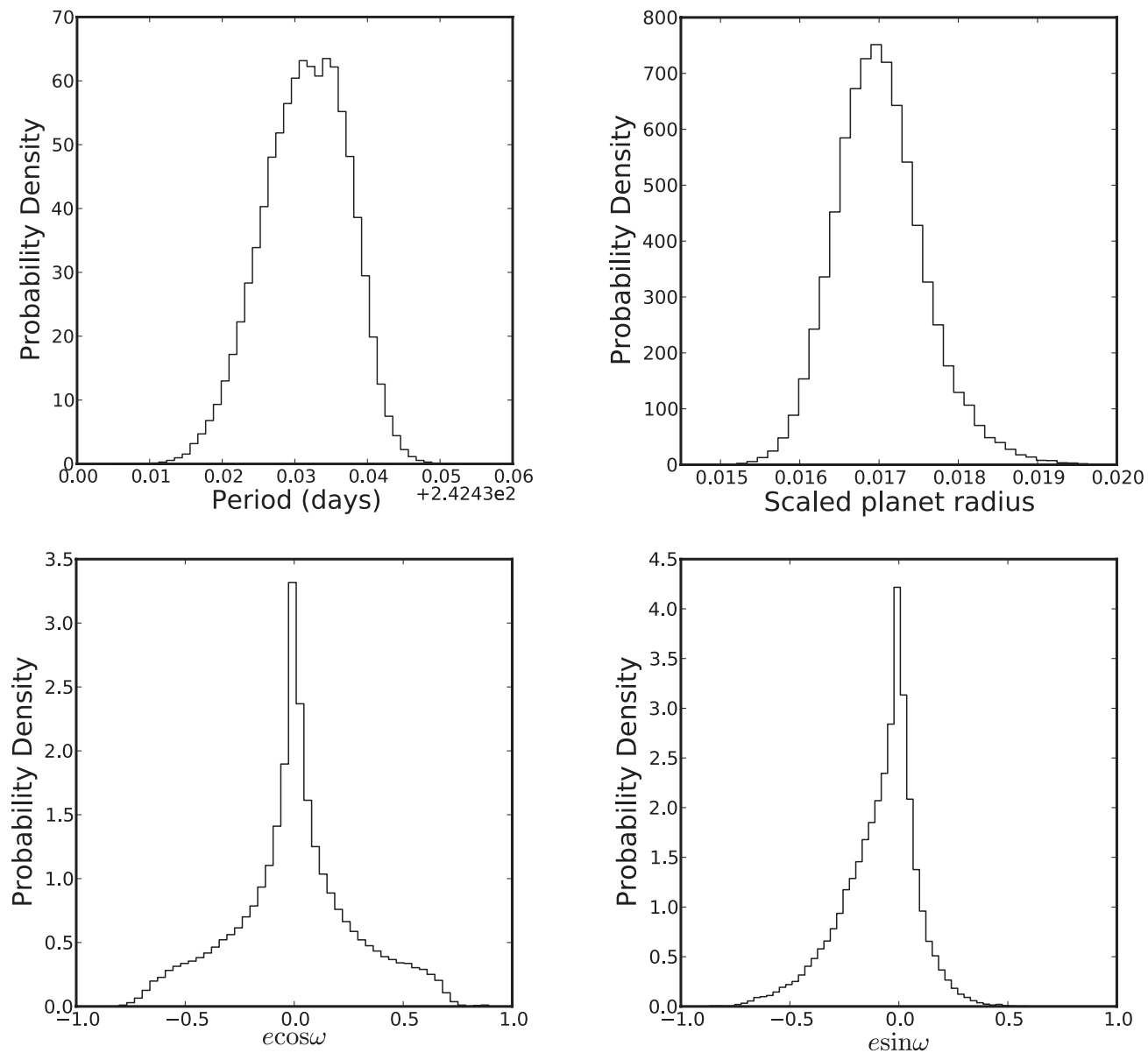

Figure 1. Probability density distributions for the MLE Kepler-69c parameters shown in Table 1 , including the orbital period (top left), $R_{p} / R_{*}$ (top right), $e$ cos $\omega$ (bottom left), and $e \cos \omega$ (bottom right).

Table 1

Kepler-69 System Parameters

\begin{tabular}{|c|c|c|c|c|c|c|}
\hline \multicolumn{2}{|c|}{ Star } & \multicolumn{5}{|c|}{ Planets } \\
\hline \multirow[t]{2}{*}{ Parameter } & \multirow[t]{2}{*}{ Value } & \multirow[t]{2}{*}{ Parameter } & \multicolumn{2}{|c|}{ MCMC Analysis } & \multicolumn{2}{|c|}{ Maximum Likelihood (MLE) } \\
\hline & & & Kepler-69b & Kepler-69c & Kepler-69b & Kepler-69c \\
\hline$T_{\text {eff }}(\mathrm{K})$ & $5638 \pm 168$ & Period (days) & $13.722341_{-0.000036}^{+0.000035}$ & $242.4613_{-0.0064}^{+0.0059}$ & $13.7223566_{-0.0000766}^{+0.0000534}$ & $242.467216_{-0.004784}^{+0.004784}$ \\
\hline $\log g$ & $4.40 \pm 0.15$ & $R_{p} / R_{*}$ & $0.02207_{-0.00018}^{+0.00023}$ & $0.0168_{-0.00052}^{+0.00052}$ & $0.02196_{-0.00016}^{+0.00104}$ & $0.01638_{-0.00038}^{+0.00192}$ \\
\hline $\operatorname{Mass}\left(M_{\odot}\right)$ & $0.810_{-0.081}^{+0.090}$ & $e \cos \omega$ & $0.02_{-0.14}^{+0.19}$ & $-0.01_{-0.16}^{+0.14}$ & $0.000076_{-0.50}^{+0.50}$ & $0.0060_{-0.58}^{+0.60}$ \\
\hline Radius $\left(R_{\odot}\right)$ & $0.93_{-0.12}^{+0.18}$ & $e \sin \omega$ & $-0.07_{-0.14}^{+0.09}$ & $-0.02_{-0.15}^{+0.08}$ & $0.000039_{-0.41}^{+0.19}$ & $0.0071_{-0.51}^{0.21}$ \\
\hline Luminosity $\left(L_{\odot}\right)$ & $0.80_{-0.22}^{+0.37}$ & $e$ & $0.16_{-0.001}^{+0.17}$ & $0.14_{-0.10}^{+0.18}$ & 0.0 (fixed) & 0.0 (fixed) \\
\hline
\end{tabular}

values is that the final Keplerian solution is not necessarily selfconsistent with respect to the orientation and ellipticity of the orbit. For example, solving $e \cos \omega$ and $e \sin \omega$ for $e$ using the MCMC median values yields $e=0.07$ and $e=0.02$ for planets $\mathrm{b}$ and $\mathrm{c}$, respectively. The Keplerian components of the orbital solution are important for the $\mathrm{HZ}$ calculations described in Section 3 because the equilibrium temperature of both planets may vary substantially during the course of a single orbit due to the eccentricity, depending on atmospheric circularization acting as a compensator. Thus, a self-consistent solution is required.

Here, we report the MLE values from the MCMC analysis. Instead of reporting the median value for each parameter independently, we report the single MLE which the MCMC converges upon. This represents both a single element in the MCMC chain and a self-consistent Keplerian solution. This solution is shown in Table 1 alongside the MCMC solution from Barclay et al. (2013a). The probability density distribution for each of the parameters from this MLE solution are shown in Figure 1. We also include the uncertainties for each of the parameters, which for a given MCMC chain is the credible interval which represents $95 \%$ of the parameter distribution. One thing to note is the relatively large uncertainties associated with the $e \cos \omega$ and $e \sin \omega$ terms. The determination of $e$ and $\omega$ are intricately linked to the impact parameter of the transit crossing and the transit duration (Kane et al. 2012a). The transit durations for eccentric and circular orbits are related by the following equation:

$$
\frac{t_{\mathrm{ecc}}}{t_{\mathrm{circ}}}=\frac{\sqrt{1-e^{2}}}{1+e \cos \left(\omega-90^{\circ}\right)} .
$$



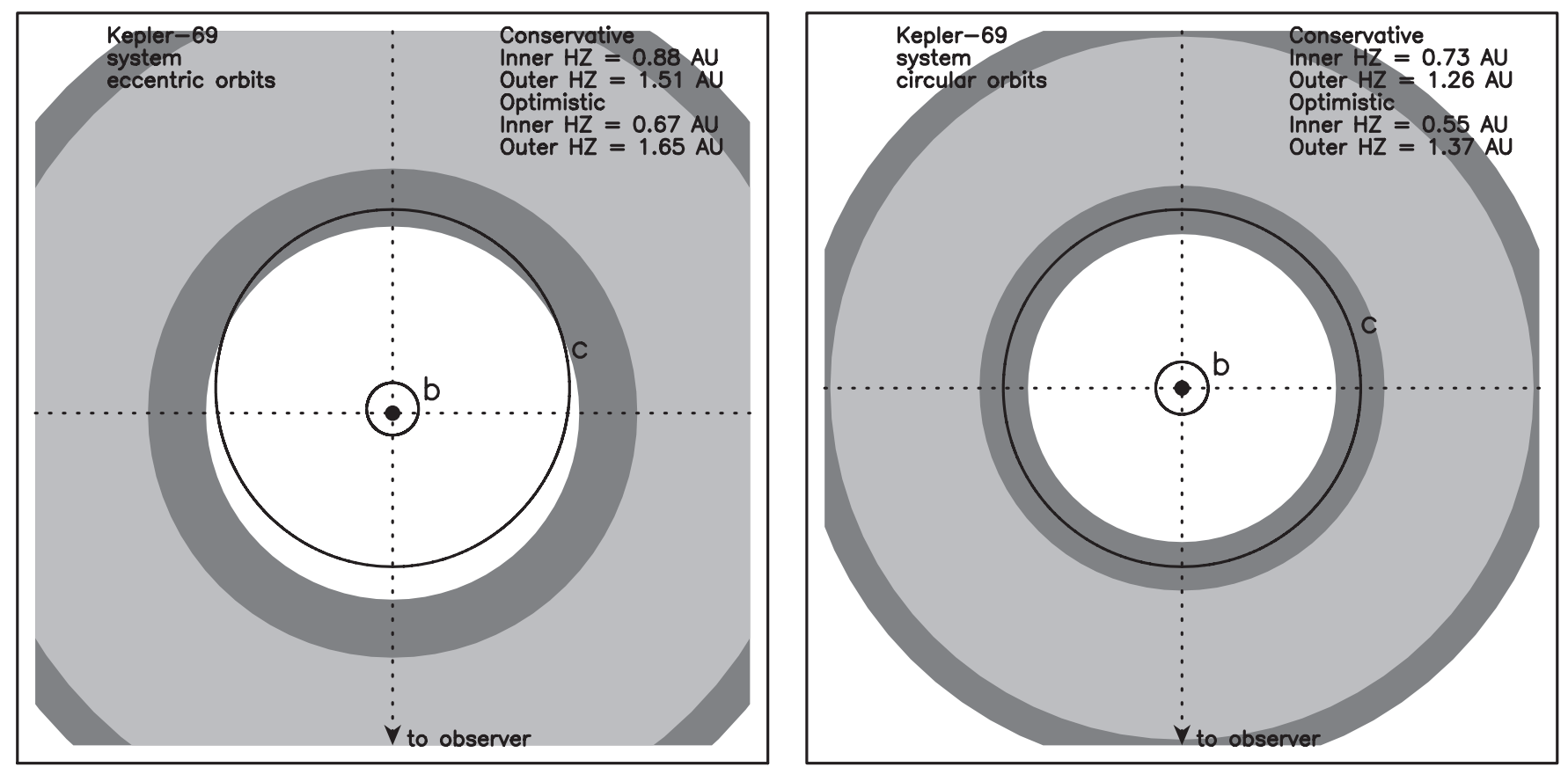

Figure 2. Calculated extent of the conservative (light gray) and optimistic (dark gray) HZ for the Kepler- 69 system along with the Keplerian orbits of the planets. Left panel: $\mathrm{HZ}$ using the stellar parameters and Barclay et al. (2013a) orbital solutions shown in Table 1. Right panel: HZ using Table 1 stellar parameters reduced by $1 \sigma$ and the circular orbital solution presented in this Letter.

The transit duration is also highly dependent upon the stellar radius which, as shown in Table 1 , is poorly determined for this star. The degeneracy between impact parameter and transit duration in determining eccentricity combined with the relatively high uncertainty in the stellar radius thus results in uncertainty associated with the values of $e$ and $\omega$. Our presented solution is consistent with a zero eccentricity. In the following sections, we investigate both circular orbits and the eccentricities found by Barclay et al. (2013a).

\section{THE SYSTEM HABITABLE ZONE}

The HZ is usually defined as the region around a star where water can exist in a liquid state on the surface of a planet with sufficient atmospheric pressure. One-dimensional climate models were utilized by Kasting et al. (1993) to quantify the inner and outer boundaries of the $\mathrm{HZ}$ for various types of main-sequence stars. These models have been used by many investigators and have been re-interpreted as analytical functions of stellar effective temperature and luminosity by several authors including Selsis et al. (2007). The work of Kasting et al. (1993) was recently replaced by the revised models of Kopparapu et al. (2013) which includes an extension of the methodology to later spectral types. These calculations are available through the Habitable Zone Gallery (Kane \& Gelino 2012b), which provides $\mathrm{HZ}$ calculations for all known exoplanetary systems.

The Kasting et al. (1993) and Kopparapu et al. (2013) approach considers conditions whereby the temperature equilibrium would sway to a runaway greenhouse effect or to a runaway snowball effect based upon the stellar effective temperature, stellar flux, and water absorption by the atmosphere. Their boundaries allow for both conservative and optimistic scenarios depending on how long it is presumed that Venus and Mars were able to retain liquid water on their surfaces. Here we use two models, referred to as the "conservative" model and the "optimistic" model. The conservative model uses the
"Runaway Greenhouse" and "Maximum Greenhouse" criteria for the inner and outer HZ boundaries, respectively. The optimistic model uses the "Recent Venus" and "Early Mars" criteria for the inner/outer HZ boundaries. These criteria are described in detail by Kopparapu et al. (2013).

Figure 2 shows the orbits of the Kepler-69 planets overlaid on the calculated HZ regions for the star. The light gray represents the conservative regions and the dark gray regions represent the extensions to the HZ from the optimistic model. The left panel uses the stellar parameters and Barclay et al. (2013a) orbital solutions as shown in Table 1. The semimajor axes are $0.094 \mathrm{AU}$ for planet $\mathrm{b}$ and $0.64 \mathrm{AU}$ for planet $\mathrm{c}$. For the conservative model, we calculate inner and outer $\mathrm{HZ}$ boundaries of $0.88 \mathrm{AU}$ and 1.51 AU, respectively. For this model, neither planet ever enters the HZ. For the optimistic model, the inner and outer HZ boundaries are $0.67 \mathrm{AU}$ and $1.65 \mathrm{AU}$, respectively. In this case, the apastron of the planet $\mathrm{c}$ orbit enters the HZ, assuming the eccentricity of 0.14 shown in Table 1 . We calculate that $43.9 \%$ of the total orbital period is spent in the $\mathrm{HZ}$ where it is slowly moving through apastron. Planets in eccentric orbits which spend only part of their orbit in the HZ have been previously investigated (Kane \& Gelino 2012c; Williams \& Pollard 2002). These studies show that habitability is not necessarily ruled out. However, a grazing encounter with the optimistic inner boundary does not qualify the planet to be considered an "HZ planet" in this model.

We further investigate a scenario which uses our MLE solution shown in Table 1 for both planets. We assume that the stellar parameters are $1 \sigma$ lower than their measured values, thus we use an effective temperature and stellar radius of $T_{\text {eff }}=5470 \mathrm{~K}$ and $R_{*}=0.81 R_{*}$, respectively. This results in the $\mathrm{HZ}$ moving inward slightly. For the conservative model, the inner and outer $\mathrm{HZ}$ boundaries are $0.73 \mathrm{AU}$ and $1.26 \mathrm{AU}$, respectively. For the optimistic model, the inner and outer HZ boundaries are $0.55 \mathrm{AU}$ and $1.37 \mathrm{AU}$, respectively. This scenario is depicted in the right panel of Figure 2, and shows that planet $\mathrm{c}$ 
may feasibly spend $100 \%$ of the orbit within the inner edge of the optimistic boundary. Recall that this uses the "Recent Venus" criteria (Kopparapu et al. 2013), which is empirically determined from an assumption that Venus once had surface liquid water but has been dry for at least the past 1 billion years (Solomon \& Head 1991). If we adopt the unmodified stellar parameters, then planet $\mathrm{c}$ is always interior to even the optimistic HZ.

There are several intrinsic properties of the planet which may move the effective $\mathrm{HZ}$ closer to the star and thus encompass the planetary orbit. The first of these is the effect of clouds. As pointed out by Kopparapu et al. (2013), $\mathrm{H}_{2} \mathrm{O}$ and $\mathrm{CO}_{2}$ cloud effects are not included in their models since threedimensional climate models are required. Attempts have been made to include specific cloud assumptions in HZ calculations, such as the work of Selsis et al. (2007). Scaling the results of Selsis et al. (2007) to the case of Kepler-69c is unlikely to be valid since they use a one-dimensional model which assumes an Earth-based atmospheric scale height which will certainly be different for the larger size of Kepler-69c. As shown by Forget \& Pierrehumbert (1997), neglecting $\mathrm{CO}_{2}$ clouds may result in an underestimation of the greenhouse effect. Generally speaking, the effect of $\mathrm{H}_{2} \mathrm{O}$ clouds is to move the inner $\mathrm{HZ}$ boundary closer to the star but $\mathrm{CO}_{2}$ clouds result in IR backscattering, thus increasing the surface temperature and moving the HZ outward. Thus the extent to which the HZ boundary moves is sensitive to the underlying assumptions regarding the composition and convection of the planetary atmosphere.

A second property of the planet which may influence the extent of the HZ is the mass. Kopparapu et al. (2013) briefly address this aspect for Mars, Earth, and super-Earth $\left(10 M_{\oplus}\right)$ planets. In particular, they examine the effect of surface gravity which influences the column depth of the atmosphere and the subsequent strength of the greenhouse effect. Their results show that a larger surface gravity will move the inner edge inward, but a lower surface gravity will move the outer edge outward. The estimated radius of Kepler-69c is $1.71 R_{\oplus}$ (Barclay et al. 2013a). Using the stellar and planetary properties shown in Table 1 and the mass/radius/flux relationships of Weiss et al. (2013), we estimate a Kepler-69c mass of $2.14 M_{\oplus}$. This results in a density of $\rho=2.36 \mathrm{~g} \mathrm{~cm}^{-3}$ and a surface gravity of 0.73 times that of the Earth. This relatively low surface gravity implies that the mass and size of the planet will have little effect on the $\mathrm{HZ}$ and may in fact move the inner HZ boundary outward.

Finally, we note that Barclay et al. (2013a) address the issue of the $\mathrm{HZ}$ by calculating the equilibrium temperature of the planet based upon incident stellar flux and assumptions regarding the Bond albedo. As pointed out by Kopparapu et al. (2013), this approach is problematic since the albedo is wavelength dependent and may lead to discrepant values for the HZ boundaries. Our analysis here shows that Kepler-69c is unlikely to be in the HZ of the host star, although it is a good candidate to be confirmed as a super-Venus.

\section{IMPLICATIONS FOR A POSSIBLE SUPER-VENUS}

Considering that Kepler-69c has a similar orbital period to that of Venus, we consider here the possibility that the planet in fact may bear a Venusian surface environment at an increased scale. A particularly compelling consideration is that of the incident flux. The solar flux received by the Earth is approximately $1365 \mathrm{~W} \mathrm{~m}^{-2}$ and the flux received by Venus is $2611 \mathrm{~W} \mathrm{~m}^{-2}$, thus Venus receives 1.91 times more flux than the Earth. Kepler-69c receives a flux of $2614 \mathrm{~W} \mathrm{~m}^{-2}$ from the host star which is also a factor of 1.91 times the flux received at Earth. This similarity in incident flux received between Venus and Kepler-69c is a primary motivator in discussing potential other similarities.

The relatively low density of the planet of $\rho=2.36 \mathrm{~g} \mathrm{~cm}^{-3}$ is similar to the Galilean moons of our solar system and indicates composition dominated by silicate and carbonate minerals rather than metals, consistent with the less than solar metallicity (Barclay et al. 2013a). Assuming that the planet formed in situ, it will have been subjected to water delivery during the late heavy bombardment, although it has been suggested by Morbidelli et al. (2000) that terrestrial planets can accrete significant water during formation without the need for cometary impacts. With such water delivery and a similar atmospheric evolution to Venus, the availability of silicates and carbonates in the crust of the planet may well have produced a thick $\mathrm{CO}_{2}$ atmosphere and subsequent runaway greenhouse warming. In addition, the non-zero eccentric solution of Barclay et al. (2013a) will help to trigger further atmospheric changes via tidal heating (Barnes et al. 2013b). Thus a more likely scenario than a habitable superEarth with liquid water is an inhospitable planetary surface with a thick $\mathrm{CO}_{2}$ atmosphere, high temperatures, and high atmospheric pressure.

While developing techniques to characterize the planets that contribute to the $\eta_{\oplus}$ population, such as photometric variability due to dynamic weather (Pallé et al. 2008), we can similarly determine how to distinguish these planets from those that comprise $\eta_{\text {Venus }}$. Although the Venusian surface is hot, the upper cloud layers are cool and so not necessarily a good indication of surface conditions. These clouds are also highly reflective, with a geometric albedo of 0.65 compared with 0.37 for the Earth. This means that a relatively large amplitude of reflected light (phase) variations for such planets may be a first indicator of a super-Venus in exosystems (Mallama 2009). In our own solar system, Venus is second only to Jupiter (43\%) in the amplitude of the phase variations (Kane \& Gelino 2013). In Figure 3 we show the calculated phase variations for the Kepler-69 system, including the effects of both planets for one complete orbital period of planet c. For this model, we have assumed a high Venusian geometric albedo and a very low albedo for the inner planet. Since the phase amplitude is proportional to $R_{p}^{2} / a^{2}$, the inner planet will overwhelmingly dominate the phase signature. Even so, the phase amplitude of planet $\mathrm{c}$ is $\sim 3.5$ times higher than Venus and thus may be a useful diagnostic in similar systems. Note that the geometric albedo and therefore the phase amplitude has a strong wavelength dependence.

A more robust method is to measure the major atmospheric components via transmission spectroscopy (Hedelt et al. 2013). The atmospheric signature of Venus has been extensively studied, and further opportunities via the recent 2004 and 2012 transits have been exploited to great effect (Hedelt et al. 2011). It is well established that Earth and Venus have many differences between their transmission spectra, the most prominent being due to the type of scattering that dominates in the UV and optical wavelengths (Ehrenreich et al. 2012). For Earth, Rayleigh scattering is the dominant effect, whereas for Venus it is Mie scattering in the cloud and haze layers that influences the shape of the spectral continuum.

Another signature of the Venusian atmosphere is the identification of the $\mathrm{CO}_{2}$ dominant atmosphere. In emission spectra, $\mathrm{CO}_{2}$ features emission peaks at 4.3 and $15 \mu \mathrm{m}$ due to the atmospheric temperature inversion (Hedelt et al. 2013). Observations of atmospheric abundances at low altitudes is difficult for Venus 


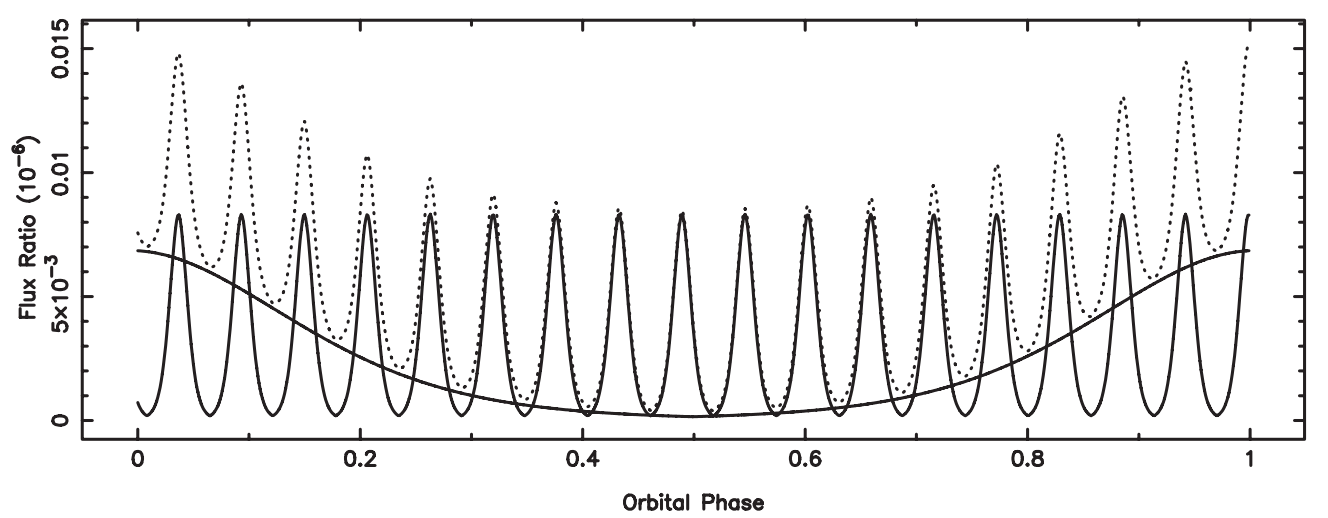

Figure 3. The model photometric flux variations due to planetary phases in the Kepler-69 system for one complete orbital period of planet c. The solid lines show the phase variations due to the individual planets and the dotted line indicates the combined effect.

since $\mathrm{H}_{2} \mathrm{SO}_{4}$ clouds are optically thick and obscure the lower atmosphere (Schaefer \& Fegley 2011; Ehrenreich et al. 2006). However, because of the very different temperature structures of the Earth and Venus atmospheres, the $\mathrm{CO}_{2}$ show significant differences in transmission spectra. As mentioned earlier, the Venusian upper atmosphere is efficiently cooled due to the strong greenhouse effect trapping the IR radiation at the surface. Thus, a lack of detectable IR $\mathrm{CO}_{2}$ emissions in the upper atmosphere may indicate the presence of a greenhouse closer to the surface which renders the planet uninhabitable. The detectability of such signatures has been investigated by Ehrenreich et al. (2012) and found to be feasible with planned instrumentation for the James Webb Space Telescope (JWST) and the European Extremely Large Telescope, although ground-based detection of $\mathrm{CO}_{2}$ bands is rendered nearly impossible due to the strong presence of the molecule in our own atmosphere. Even so, there are indeed future instrumentation opportunities that will remove the ambiguity when attempting to distinguish between super-Earth and super-Venus atmosphere analogs.

\section{CONCLUSIONS}

The extraordinary diversification of the exoplanetary field is enabling us to finally place our solar system in context. The frequency of Jupiter analogs and Earth analogs are key components to understanding the precise nature of that context. However, as the sensitivity of current detection techniques reaches into the terrestrial-size regime, we also need to be able to distinguish between Earth and Venus analogs in order to place our habitability in context. Thus, the key question of how common Earth-like planets are relies on also understanding how frequently terrestrial planets diverge into Venusian-type evolutionary pathways which may place heavy constraints upon habitability prospects. The Kepler mission is already revealing many planets which fall into the terrestrial-size planetary regime and, as we have shown here, there are cases where the properties of the system straddle current HZ boundary calculations. The case of the Kepler-69 system is particularly interesting because the host star is only slightly less massive than our own Sun, thus providing an easier comparison to the $\mathrm{HZ}$ and planets or our solar system. It is a considerable hindrance to our studies of largerthan-Earth terrestrial planets that our system contains no such objects. However, considering the high occurrence rate of these types of planets, it is likely that these will dominate exoplanet atmospheric studies well into the next decade. Atmospheric characterization of large terrestrial planets has a productive future ahead of it with the anticipated discoveries of the
Transiting Exoplanet Survey Satellite and subsequent followup with the JWST. The detection of Venusian-type atmospheres at a variety of orbital periods and planetary masses will create a picture of where the divergence between Earth and Venus originates.

The authors would like to thank David Ciardi, Lauren Weiss, and the anonymous referee for productive feedback. This work has made use of the Habitable Zone Gallery at hzgallery.org.

\section{REFERENCES}

Barclay, T., Burke, C. J., Howell, S. B., et al. 2013a, ApJ, 768, 101 Barclay, T., Rowe, J. F., Lissauer, J. J., et al. 2013b, Natur, 494, 452 Barnes, R., Mullins, K., Goldblatt, C., et al. 2013, AsBio, 13, 225 Batalha, N. M., Rowe, J. F., Bryson, S. T., et al. 2013, ApJS, 204, 24 Borucki, W. J., Agol, E., Fressin, F., et al. 2013, Sci, in press (arXiv:1304.7387) Borucki, W. J., Koch, D. G., Basri, G., et al. 2011a, ApJ, 728, 117 Borucki, W. J., Koch, D. G., Basri, G., et al. 2011b, ApJ, 736, 19 Borucki, W. J., Koch, D. G., Batalha, N., et al. 2012, ApJ, 745, 120

Dressing, C. D., \& Charbonneau, D. 2013, ApJ, 767, 95

Eastman, J., Gaudi, B. S., \& Agol, E. 2013, PASP, 125, 83

Ehrenreich, D., Tinetti, G., Lecavelier Des Etangs, A., Vidal-Madjar, A., \& Selsis, F. 2006, A\&A, 448, 379

Ehrenreich, D., Vidal-Madjar, A., Widemann, T., et al. 2012, A\&A, 537, L2

Foreman-Mackey, D., Hogg, D. W., Lang, D., \& Goodman, J. 2013, PASP, 125,306

Forget, F., \& Pierrehumbert, R. T. 1997, Sci, 278, 1273

Hedelt, P., Alonso, R., Brown, T., et al. 2011, A\&A, 533, A136

Hedelt, P., von Paris, P., Godolt, M., et al. 2013, A\&A, 553, A9

Howard, A. W., Marcy, G. W., Bryson, S. T., et al. 2012, ApJS, 201, 15

Kane, S. R., Ciardi, D. R., Gelino, D. M., \& von Braun, K. 2012a, MNRAS, 425,757

Kane, S. R., \& Gelino, D. M. 2012b, PASP, 124, 323

Kane, S. R., \& Gelino, D. M. 2012c, AsBio, 12, 940

Kane, S. R., \& Gelino, D. M. 2013, ApJ, 762, 129

Kane, S. R., \& von Braun, K. 2008, ApJ, 689, 492

Kasting, J. F., Whitmire, D. P., \& Reynolds, R. T. 1993, Icar, 101, 108

Kopparapu, R. K. 2013, ApJL, 767, L8

Kopparapu, R. K., Ramirez, R., Kasting, J. F., et al. 2013, ApJ, 765, 131

Mallama, A. 2009, Icar, 204, 11

Morbidelli, A., Chambers, J., Lunine, J. I., et al. 2000, M\&PS, 35, 1309

Pallé, E., Ford, E. B., Seager, S., Montañés-Rodríguez, P., \& Vazquez, M. 2008, ApJ, 676, 1319

Petigura, E. A., Marcy, G. W., \& Howard, A. W. 2013, ApJ, in press (arXiv:1304.0460)

Schaefer, L., \& Fegley, B. 2011, ApJ, 729, 6

Selsis, F., Kasting, J. F., Levrard, B., et al. 2007, A\&A, 476, 1373

Solomon, S. C., \& Head, J. W. 1991, Sci, 252, 252

Traub, W. 2012, ApJ, 745, 20

Valenti, J. A., \& Fischer, D. A. 2005, ApJS, 159, 141

Weiss, L. M., Marcy, G. W., Rowe, J. F., et al. 2013, ApJ, 768, 14

Williams, D. M., \& Pollard, D. 2002, IJAsB, 1, 61 